\title{
Metodología para seleccionar oportunidades de mejora continua en promociones de viviendas
}

\author{
Methodology to select continuous improvement opportunities \\ in housing blocks
}

$\underline{\text { P. Del Solar }}^{(*)}$, M. Del Río ${ }^{(*)}$

RESUMEN

La mejora continua debería estar presente siempre en las empresas, dispongan o no de sistemas de gestión conforme a la Norma UNE EN ISO 9001:2008. Sin embargo, su aplicación en el sector de la construcción es especialmente difícil debido a las características particulares del mismo (1). Este articulo describe cómo aplicar cuatro de las siete herramientas estadísticas básicas de la mejora continua (Hoja de recogida de datos, Estratificación, Histograma y Diagrama de Pareto) para seleccionar oportunidades de mejora, dentro de la fase de planificación de un proyecto de mejora continua que tenga como objetivo reducir los defectos de construcción en edificios de viviendas detectados en la fase de pre-entrega. Concluyendo que los tres oficios en los que convendría actuar para reducir de manera significativa los fallos de construcción en la fase de pre-entrega son: Carpintería de Madera, Revestimientos Cerámicos e Instalación de Electricidad.

Palabras clave: Mejora continua; calidad; gestión; fallos de construcción; defectos de construcción.

\section{ABSTRACT}

Continuous improvement should always be present in business, have they or not management systems on hand according to the UNE EN ISO 9001:2008 Standard. However, its application in the construction sector is especially hard due to the particular characteristics of this business (1). This paper focuses on how to apply four of the seven basic statistical continuous improvement tools (Data collection sheet, Stratification, Histogram and Pareto Diagram) in order to select improvement opportunities within the planification phase of a continuous improvement project whose aims is the reduction of the construction failures in housing blocks which would be detected during the pre-delivery phase. The conclusion is that the three works in which it would be convenient to act in order to reduce significantly the construction failures during the pre-delivery phase are: Wood Carpentry, Ceramic Tile and Electricity.

Keywords: Continuous improvement; quality; management; construction failures; construction defects.

(*) Universidad Politécnica de Madrid (España).

Persona de contacto/Corresponding author: patricia.delsolar@upm.es (P. Del Solar)

Cómo citar este artículo/Citation: Del Solar, P., Del Río, M. (2015). Metodología para seleccionar oportunidades de mejora continua en promociones de viviendas. Informes de la Construcción, 67(538): e073, doi: http://dx.doi.org/10.3989/ic.13.142.

Licencia / License: Salvo indicación contraria, todos los contenidos de la edición electrónica de Informes de la Construcción se distribuyen bajo una licencia de uso y distribución Creative Commons Reconocimiento no Comercial 3.o. España (cc-by-nc). 


\section{INTRODUCCIÓN}

En un mundo donde la globalización es una realidad y donde la competencia es cada vez mayor, las empresas deben ser capaces de garantizar la calidad de sus productos y servicios.

Y para ello es preciso que cuenten con sistemas de gestión empresarial que faciliten y aseguren que todos los departamentos y el personal de la empresa trabajan bajo unos estándares determinados, de manera que los procesos y procedimientos de la organización se encuentren permanentemente supervisados para asegurar que se ofrece la máxima calidad a los clientes.

Aunque son muchos los sistemas de gestión que existen en el mercado, en el sector de la construcción y en concreto en nuestro país, los más extendidos son los que se certifican conforme al grupo de Normas ISO 9000.

Uno de los pilares fundamentales que defienden el grupo de Normas ISO 9000 para garantizar la eficacia y eficiencia de los Sistemas de Gestión de la Calidad es la Mejora Continua.

Se define Proyecto de Mejora Continua como: «Proyecto seleccionado por los órganos competentes de la organización, cuyo objetivo es la eliminación o reducción de la diferencia identificada entre la situación deseada y la situación real relativa a una o más oportunidades de mejora» (2).

Una de las formas de articular un Proyecto de Mejora Continua es aplicar el Círculo de Calidad de Deming (PDCA), o sus variantes, a determinados procesos o actividades de la empresa, utilizando todas o alguna de las herramientas estadísticas básicas de la calidad.

Las fases del Círculo de Calidad son las siguientes:

- P: Plan - Planificar antes de actuar

- D: Do - Aplicar las medidas estudiadas

- C: Check - Verificar el resultado de las medidas aplicadas

- A: Act - Repetir si el resultado no ha sido positivo o Normalizar si el resultado ha sido positivo

Las siete herramientas básicas de la calidad definidas por Ishikawa (4), que son reconocidas por todos los autores en la materia como herramientas fundamentales para la mejora continua, son:

1. Los Diagramas de Pareto

2. Los Diagramas Causa-Efecto

3. Estratificación

4. Hoja de comprobación (o de recogida de datos)

5. Histogramas

6. Diagramas de dispersión

7. Gráficos de control

Para llevar a cabo el proceso de Mejora Continua, y dentro de la fase de Planificación, es preciso empezar detectando y analizando donde se está fallando y qué se puede mejorar, ya que sólo conociendo los fallos y analizando sus causas se pueden tomar acciones preventivas que eviten que vuelvan a repetirse.
Por tanto, la primera actividad que debería llevarse a cabo es la inspección del producto, el edificio de viviendas, y realizar un listado de los defectos encontrados, datos que una vez analizados servirán para establecer las medidas a aplicar en los planes de mejora.

Desafortunadamente, la experiencia práctica confirma que, en la mayoría de los casos, esta actividad o no se ejecuta, o no se realiza de forma eficaz en el sector de la construcción de viviendas en España, principalmente debido a que las particularidades del mismo hacen especialmente compleja su implantación, como explica A. García Meseguer (1).

\section{OBJETIVOS}

El objetivo del trabajo que aquí se presenta es determinar sobre qué oficios es preciso actuar en primer lugar para conseguir una disminución significativa de los defectos que generalmente se presentan en la fase previa a la entrega de las viviendas a sus futuros usuarios.

Para ello se trabaja en la fase de Planificación del Círculo de Calidad, aplicando cuatro de las siete herramientas básicas de la calidad: Hoja de recogida de datos, Estratificación, Histograma y Diagrama de Pareto.

\section{ANTECEDENTES}

A lo largo de los últimos años algunos autores han trabajado sobre la Gestión de la Calidad en el ámbito de la Edificación en España. Unos han puesto de manifiesto las dificultades que entraña el sector de la construcción (1), otros defienden la importancia de implementar Sistemas de Gestión de la Calidad, no sólo en las empresas constructoras, también en las empresas de servicios de Arquitectura Técnica (3).

También ponen de manifiesto la importancia de la Mejora Continua, proceso en el que todos los miembros de la organización deben estar implicados, siendo fundamental que el trabajador que está en primera línea de producción obtenga la formación adecuada para llevarlo a cabo (4) y que esté respaldado por la alta dirección (3).

Y es precisamente la alta dirección la que tiene la responsabilidad de buscar continuamente la mejora de los procesos de gestión y producción de sus empresas (2), (5).

Las oportunidades de mejora se pueden detectar de forma interna o externa. De forma interna sería a través de los mecanismos de control de la organización, como por ejemplo la inspección del producto, como se muestra en este trabajo.

Y las estrategias para implementar proyectos de mejora son varias, como por ejemplo a través de un sistema de «No Conformidades», mediante «Encuestas de satisfacción» o aplicando el «Círculo de Calidad de Deming: PDCA», opción elegida para este estudio, entre otras.

Por otra parte, son varios los autores que estudian los defectos de construcción, generalmente centrados en fallos que han aparecido en los edificios como consecuencia de su uso, falta de mantenimiento o deterioro causado por las condiciones atmosféricas. Como por ejemplo: 
- Fallos en la adherencia de los revestimientos cerámicos exteriores (6) y defectos en los locales húmedos (7). Estos estudios ofrecen listados de los defectos más repetidos y las causas más comunes de los mismos.

- Defectos en tejados (8) (9), en revestimientos de piedra natural (10), pastas de yeso para particiones y techos (11), revestimientos cerámicos (12) y revestimientos cerámicos de fachadas (13). Estos autores proponen una metodología, sistema de inspección y diagnóstico de defectos, para la toma de datos a través de un análisis visual de los inmuebles, clasificación de las deficiencias y análisis a través de matrices de correlación entre los defectos y la causa probable de los mismos, y los defectos y las técnicas de reparación. Aportando además «lecciones aprendidas» en base a los resultados obtenidos, para su aplicación en futuros proyectos. Las causas de los defectos las resumen en las siguientes categorías: «Errores de diseño»; «Errores de ejecución»; «Problemas con los materiales»; «Acciones mecánicas externas»; "Acciones medioambientales»; «Falta de mantenimiento» $\mathrm{y}$ «Cambios en las condiciones previstas inicialmente».

- Anomalías en los revestimientos de piedra natural (14). Los autores en este caso proponen un sistema de predicción para la vida útil de los mismos.

- Desperfectos en la envolvente de los edificios (15). Este estudio además propone una metodología para la toma de datos que, partiendo de una inspección visual de los edificios aporta unas escalas de evaluación basadas en el estado en el que se encuentran los elementos constructivos, desde «Excepcional sin necesidad de ninguna intervención» hasta «inaceptable demolición/sustitución». También aporta ejemplos de fichas en las que relaciona el sistema constructivo con su función, el modo de fallo, las causas probables del fallo, efectos directos y efectos indirectos relacionados con la deficiencia detectada.

- Estandarización de los defectos en viviendas (16). Este articulo habla sobre las desviaciones económicas y temporales que producen los defectos en los proyectos. También describe una metodología para la toma de datos sobre defectos y el análisis de los mismos.

Sin embargo no se ha encontrado ningún estudio que analice los fallos en los edificios de viviendas en el momento previo a su entrega a los usuarios para detectar oportunidades de mejora.

\section{METODOLOGÍA}

Para desarrollar el objetivo que se plantea en este artículo se trabaja en la fase de planificación de un Proyecto de Mejora
Continua basado en el Círculo de Calidad de Deming (PDCA). Como nos explica el Dr. Isikawa (5) (17) «el 95\% de los problemas de una empresa se pueden resolver con las siete herramientas del control de calidad». Para el estudio que aquí se presenta empleamos cuatro de las herramientas estadísticas básicas definidas por el Dr. Isikawa (5) (17): Hoja de recogida de datos, Estratificación, Histograma, Diagrama de Pareto, ya que son las herramientas que mejor resuelven las actividades a desarrollar en esta fase.

Los pasos seguidos y las herramientas aplicadas son las siguientes:

1. Recogida de datos: Se procede a la inspección de 625 viviendas, repartidas en siete obras de edificación, encontrándose un total de 66.445 incidencias que quedan registradas en la Plantilla de toma de datos. Tabla 3. "La hoja de recogida de datos es el soporte indispensable sobre el que plasmar los datos de los que tenemos necesidad» (18).

2. Estratificación: Se clasifican cada una de las incidencias según el oficio responsable de su reparación. Tabla 3. La estratificación consiste en «la subdivisión de los datos recogidos en una serie de grupos homogéneos que permiten una mejor comprensión del fenómeno que se está analizando» (18).

3. Estudio y análisis de los datos: Se representa gráficamente la frecuencia de las incidencias según el oficio responsable de realizar la reparación y se establecen las prioridades de actuación. Histograma y Diagrama de Pareto. El Diagrama de Pareto aporta información para «determinar la frecuencia o la importancia relativa de diferentes problemas o causas» y ayuda a "concentrarse en cuestiones vitales ordenándolas en términos de importancia» (19).

Las obras de edificación de viviendas inspeccionadas han sido ejecutadas por la empresa constructora ARPADA, S.A.

Todas ellas se encontraban en la fase final de ejecución, contando en su mayoría con el Certificado Final de Obra, estando pendientes únicamente de las reparaciones y remates del periodo previo a la entrega de las viviendas a los futuros usuarios.

Las Tablas 1 y 2 resumen la descripción de las siete obras.

Los datos fruto de la inspección se recogen en unos listados de incidencias (Hoja de recogida de datos) que contienen los siguientes campos:

- Nombre de la obra

- Portal

- Piso

Tabla 1. Descripción de las obras inspeccionadas.

\begin{tabular}{|c|c|c|c|c|c|c|c|c|c|c|}
\hline \multirow{2}{*}{ Fase } & \multirow{2}{*}{ Obra } & \multirow{2}{*}{ Localización } & \multirow{2}{*}{$\mathrm{N}^{0}$ viviendas } & \multicolumn{5}{|c|}{$\mathrm{N}^{0}$ dormitorios } & \multirow{2}{*}{ No Incidencias } & \multirow{2}{*}{ No Inc. / Viv. } \\
\hline & & & & 5 & 4 & 3 & 2 & $\mathbf{1}$ & & \\
\hline \multirow{7}{*}{1} & 01 & Alcobendas & 118 & $\mathrm{O}$ & $\mathrm{O}$ & 41 & 75 & 2 & 16.670 & 141,27 \\
\hline & 02 & Móstoles & 100 & 0 & o & $\mathrm{O}$ & 78 & 22 & 10.033 & 100,33 \\
\hline & 03 & Vicálvaro & 73 & $\mathrm{O}$ & $\mathrm{O}$ & 15 & 21 & 37 & 7.997 & 109,55 \\
\hline & 04 & Navalcarnero & 46 & $\mathrm{O}$ & $\mathrm{O}$ & 0 & 43 & 3 & 6.195 & 134,67 \\
\hline & 05 & Alcorcón & 112 & $\mathrm{O}$ & $\mathrm{O}$ & 110 & 2 & 0 & 11.235 & 100,31 \\
\hline & 06 & Móstoles 2 & 66 & $\mathrm{O}$ & 19 & 46 & 1 & $\mathrm{O}$ & 6.340 & 96,06 \\
\hline & 07 & Alcorcón 2 & 110 & 0 & $\mathrm{O}$ & 105 & 5 & $\mathrm{O}$ & 7.975 & 72,50 \\
\hline \multicolumn{3}{|l|}{ Total } & 625 & $\mathbf{0}$ & 19 & $\mathbf{3 1 7}$ & 225 & 64 & 66.445 & 106,31 \\
\hline
\end{tabular}


Tabla 2. Resumen Memoria de Calidades de las obras inspeccionadas.

\begin{tabular}{|c|c|c|c|c|c|c|c|}
\hline \multirow{2}{*}{ Resumen Memoria de Calidades } & \multicolumn{7}{|c|}{ Obras } \\
\hline & o1 & 02 & 03 & 04 & 05 & 06 & o7 \\
\hline Estructura de hormigón & $\mathrm{x}$ & $\mathrm{x}$ & $\mathrm{x}$ & $\mathrm{x}$ & $\mathrm{x}$ & $\mathrm{x}$ & $\mathrm{x}$ \\
\hline Fachada de ladrillo visto & $\mathrm{x}$ & $\mathrm{x}$ & $\mathrm{x}$ & $\mathrm{x}$ & $\mathrm{x}$ & $\mathrm{x}$ & $\mathrm{x}$ \\
\hline \multicolumn{8}{|l|}{ Tabiquería: } \\
\hline Ladrillo cerámico & - & $\mathrm{x}$ & $\mathrm{x}$ & $\mathrm{x}$ & $\mathrm{x}$ & $\mathrm{x}$ & - \\
\hline Pladur & $\mathrm{x}$ & - & - & - & - & - & $\mathrm{x}$ \\
\hline \multicolumn{8}{|l|}{ Revestimiento de suelos: } \\
\hline Tarima en dormitorios y salones & $\mathrm{x}$ & $\mathrm{x}$ & $\mathrm{x}$ & - & - & $\mathrm{x}$ & - \\
\hline Cerámico en dormitorios y salones & - & - & - & $\mathrm{x}$ & $\mathrm{x}$ & - & $\mathrm{x}$ \\
\hline Cerámico en cuartos húmedos & $\mathrm{x}$ & $\mathrm{x}$ & $\mathrm{x}$ & $\mathrm{x}$ & $\mathrm{x}$ & $\mathrm{x}$ & $\mathrm{x}$ \\
\hline \multicolumn{8}{|l|}{ Revestimiento de paredes: } \\
\hline Pintura acabado liso & $\mathrm{x}$ & $\mathrm{x}$ & - & - & - & $\mathrm{x}$ & $\mathrm{x}$ \\
\hline Pintura acabado en gota & - & - & $\mathrm{x}$ & $\mathrm{x}$ & $\mathrm{x}$ & - & - \\
\hline Alicatado cerámico cuartos húmedos & $\mathrm{x}$ & $\mathrm{x}$ & $\mathrm{x}$ & $\mathrm{x}$ & $\mathrm{x}$ & $\mathrm{x}$ & $\mathrm{x}$ \\
\hline Mármol en Baño principal & - & - & $\mathrm{x}$ & - & - & - & - \\
\hline Carpintería exterior de aluminio & $\mathrm{x}$ & $\mathrm{x}$ & $\mathrm{x}$ & $\mathrm{x}$ & $\mathrm{x}$ & $\mathrm{x}$ & $\mathrm{x}$ \\
\hline \multicolumn{8}{|l|}{ Carpintería interior: } \\
\hline Madera barnizada & $\mathrm{x}$ & - & $\mathrm{x}$ & $\mathrm{x}$ & $\mathrm{x}$ & $\mathrm{x}$ & $\mathrm{x}$ \\
\hline Madera lacada & - & $\mathrm{x}$ & - & - & - & - & - \\
\hline \multicolumn{8}{|l|}{ Techos: } \\
\hline Yeso & $\mathrm{x}$ & $\mathrm{x}$ & $\mathrm{x}$ & $\mathrm{x}$ & $\mathrm{x}$ & $\mathrm{x}$ & $\mathrm{x}$ \\
\hline Pladur & - & - & - & - & - & - & $\mathrm{x}$ \\
\hline Escayola & $\mathrm{x}$ & $\mathrm{x}$ & $\mathrm{x}$ & $\mathrm{x}$ & $\mathrm{x}$ & $\mathrm{x}$ & - \\
\hline Placas con perfilería vista & - & - & $\mathrm{x}$ & - & - & - & - \\
\hline \multicolumn{8}{|l|}{ Instalaciones: } \\
\hline Fontanería & $\mathrm{x}$ & $\mathrm{x}$ & $\mathrm{x}$ & $\mathrm{x}$ & $\mathrm{x}$ & $\mathrm{x}$ & $\mathrm{x}$ \\
\hline Calefacción radiadores & $\mathrm{x}$ & $\mathrm{x}$ & $\mathrm{x}$ & $\mathrm{x}$ & $\mathrm{x}$ & $\mathrm{x}$ & $\mathrm{x}$ \\
\hline Electricidad & $\mathrm{x}$ & $\mathrm{x}$ & $\mathrm{x}$ & $\mathrm{x}$ & $\mathrm{x}$ & $\mathrm{x}$ & $\mathrm{x}$ \\
\hline Telecomunicaciones & $\mathrm{x}$ & $\mathrm{x}$ & $\mathrm{x}$ & $\mathrm{x}$ & $\mathrm{x}$ & $\mathrm{x}$ & $\mathrm{x}$ \\
\hline Domótica & $\mathrm{x}$ & - & - & - & - & - & - \\
\hline Aire acondicionado Split & - & - & - & - & - & - & - \\
\hline Aire acondicionado Conductos & - & - & $\mathrm{x}$ & $\mathrm{x}$ & - & - & - \\
\hline Mobiliario de cocina & $\mathrm{x}$ & $\mathrm{x}$ & $\mathrm{x}$ & $\mathrm{x}$ & - & - & - \\
\hline
\end{tabular}

- Letra

- Localización de la incidencia (estancia dentro de la vivienda)

- Descripción de la incidencia

- Oficio que debe realizar la reparación

La Tabla 3 muestra un extracto de los listados de incidencias.

\section{RESULTADOS}

Los datos obtenidos se estudian y analizan a través de las herramientas Histograma y Diagrama de Pareto.

El Histograma resultante de la estratificación por oficio responsable de su reparación del conjunto de las siete obras, ordenado por frecuencia descendente se muestra en la Figura 1.

Se compara el resultado del conjunto de los datos con los parciales de cada una de las obras, para ello se elabora el histograma de incidencias por oficio responsable de su reparación, pero en lugar de ordenarlo por frecuencia descendente lo realizamos en el mismo orden que el general que engloba las siete obras, de manera que se pueda observar si cada obra individualmente se comporta como el conjunto. La Figura 2 muestra el histograma de una de las obras a modo de ejemplo.
Visualmente se aprecia claramente con los histogramas de cada una de las obras que no siguen la misma frecuencia de ocurrencia para cada oficio, lo que parece lógico, ya que cada obra dispone de diferentes empresas subcontratistas para cada oficio, y a su vez cada empresa subcontratista cuenta con sus propios trabajadores, por lo que éstos patrones se ven influenciados por el buen hacer propio de las personas que intervienen físicamente realizando los trabajos de su especialidad.

Sin embargo, el grueso de incidencias se concentra claramente en unos pocos oficios, comunes para todas las obras, a pesar de no mantener el orden de frecuencia. Estos oficios, que en conjunto concentran el grueso de las incidencias detectadas y que son comunes a todas las obras son:

- Pintura

- Carpintería de Madera

- Limpieza de Obra

- Revestimientos Cerámicos

A este grupo le sigue en volumen de incidencias en todas las obras, a pesar de que para las obras 05 Alcorcón, 06 Móstoles 2 y 07 Alcorcón 2 no entran dentro de los oficios que concentran el 8o\% del total de incidencias: 
Tabla 3. Ejemplo de Hoja de recogida de datos que incluye la estratificación de las incidencias.

\begin{tabular}{|l|c|c|c|l|l|l|}
\hline OBRA & PORTAL & PISO & LETRA & LOC. & \multicolumn{1}{|c|}{ OFICIO } & \multicolumn{1}{|c|}{ DESCRIPCION INCIDENCIA } \\
\hline 01 Alcobendas & 1 & o & A & Baño 2 & Inst. Fontanería & $\begin{array}{l}\text { Ajustar/enrrasar mecanismos de cisterna y se quedan } \\
\text { pillados al pulsarlos }\end{array}$ \\
\hline o1 Alcobendas & 1 & o & A & Baño 2 & Rev. Cerámicos & $\begin{array}{l}\text { Recibir y retacar toma de agua del inodoro. Se } \\
\text { mueve. El taladro del azulejo es excesivo y no lo cubre } \\
\text { el embellecedor }\end{array}$ \\
\hline 01 Alcobendas & 1 & o & A & Baño 2 & Sanitarios y grifería & Arañazo en parte frontal de cisterna \\
\hline o1 Alcobendas & 1 & o & A & Baño 2 & Limpieza de obra & $\begin{array}{l}\text { Restos de pintura en asiento e inodoro y restos de } \\
\text { cinta de embalar }\end{array}$ \\
\hline o1 Alcobendas & 1 & o & A & Baño 2 & Sanitarios y grifería & Arañazos negros en asiento e inodoro \\
\hline 01 Alcobendas & 1 & o & A & {$[6]$ baño 2 } & Inst. Fontanería & Grifo bidé con poca presión \\
\hline o1 Alcobendas & 1 & o & A & [6] baño 2 & Rev. Cerámicos & $\begin{array}{l}\text { Recibir y retacar toma de agua de la derecha del bidé. } \\
\text { El taladro del azulejo es excesivo, no se cubre con el } \\
\text { embellecedor }\end{array}$ \\
\hline
\end{tabular}

- Carpintería de Aluminio

- Instalación de Electricidad

En dos obras concretas aparecen dos oficios dentro del grupo que concentra el 80\% del total de incidencias:

- Rodapié, en la obra o1 Alcobendas

- Mobiliario de Cocina, en la obra 03 Vicálvaro

Se trata de casos aislados que no responden ni al conjunto de los datos ni se acercan a cada obra particular, por lo que no son representativas.

Por lo que se puede concluir hasta el momento que existen seis oficios que agrupan el grueso de incidencias en todas las obras en conjunto y de forma particular en cada una de ellas, siendo los siguientes:

- Pintura

- Carpintería de Madera

- Limpieza de Obra
- Revestimientos Cerámicos

- Carpintería de Aluminio

- Instalación de Electricidad

En las reuniones mantenidas con el departamento de Calidad y de Postventa de la empresa ARPADA, S.A. se decide tratar de forma independiente las incidencias detectadas en Pintura y en Limpieza de obra, por los siguientes motivos:

- Pintura: Se establece como protocolo de actuación para las obras de la constructora realizar la última mano de pintura una vez completados todos los repasos del resto de oficios.

La experiencia del equipo de Postventa confirma que en muchas ocasiones es preciso volver a pintar paños completos una vez finalizados los repasos por desperfectos ocasionados durante esta fase de obra (fundamentalmente golpes, roces y defectos de pintura mal reparados).

Posponiendo la ejecución de la última mano de pintura se consigue reducir los costes de reparación de esta

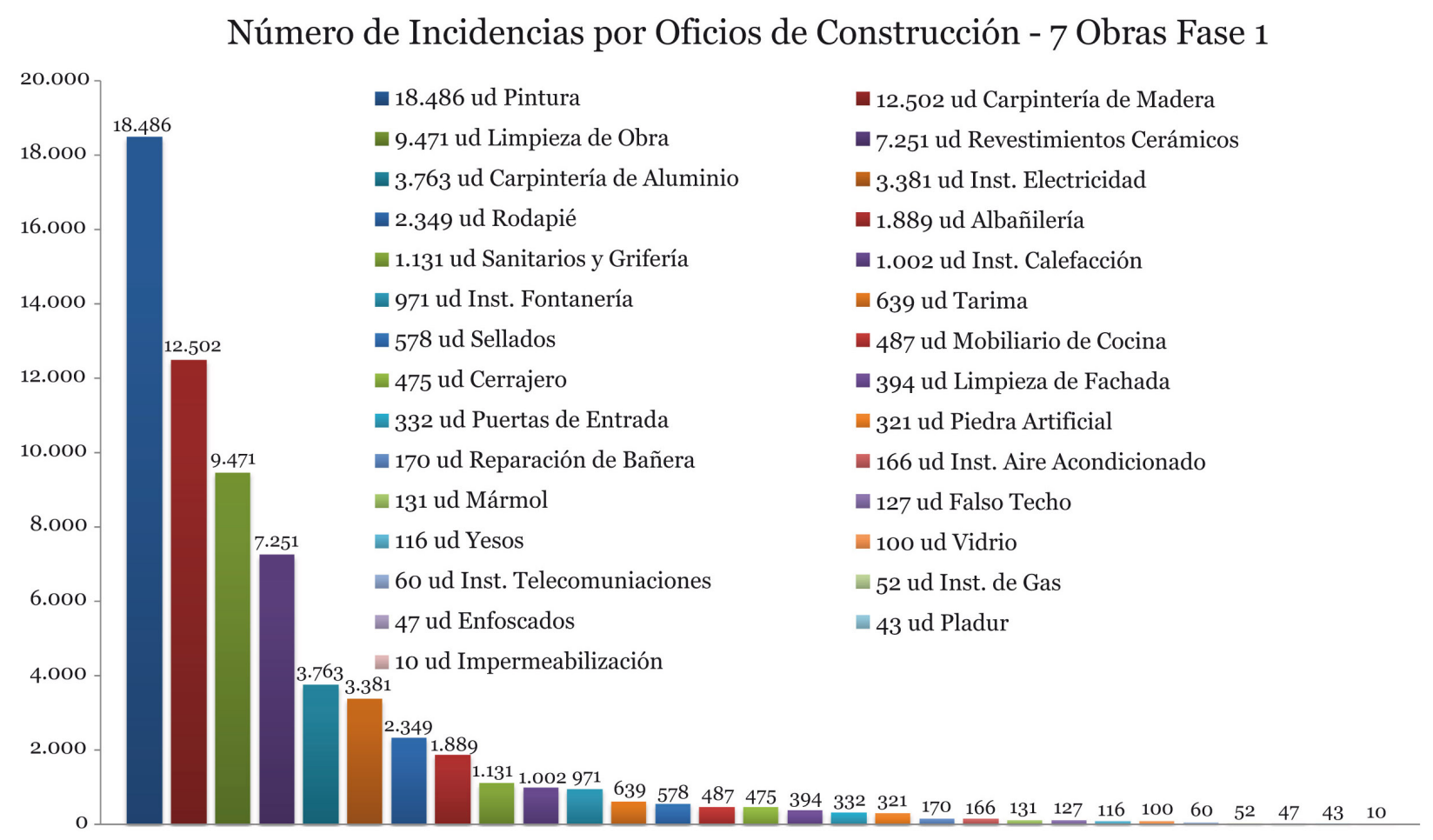

Figura 1. Histograma «Número de Incidencias por Oficios de Construcción». 


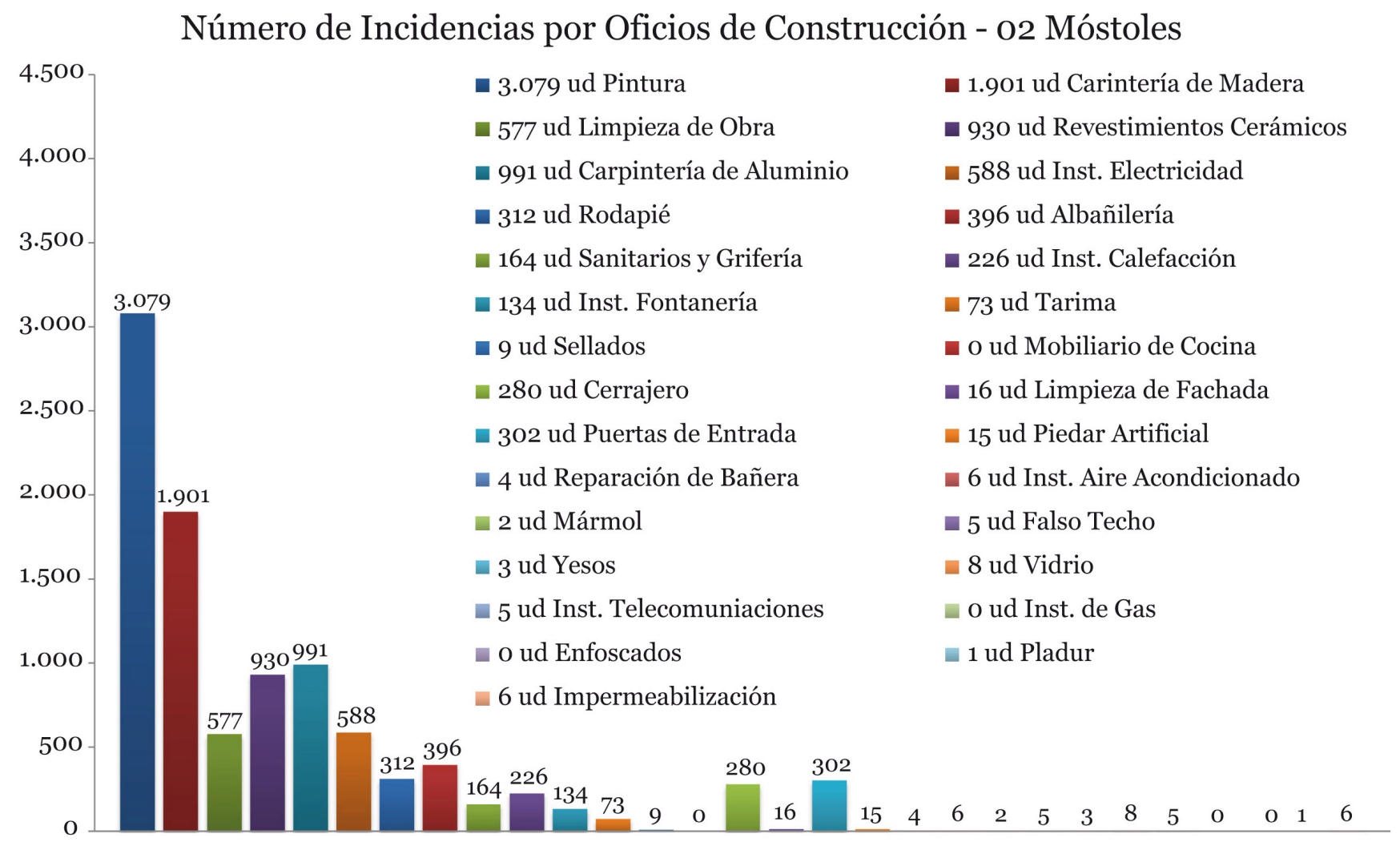

Figura 2. Histograma «Número de Incidencias por Oficios de Construcción - Obra 02 Móstoles».

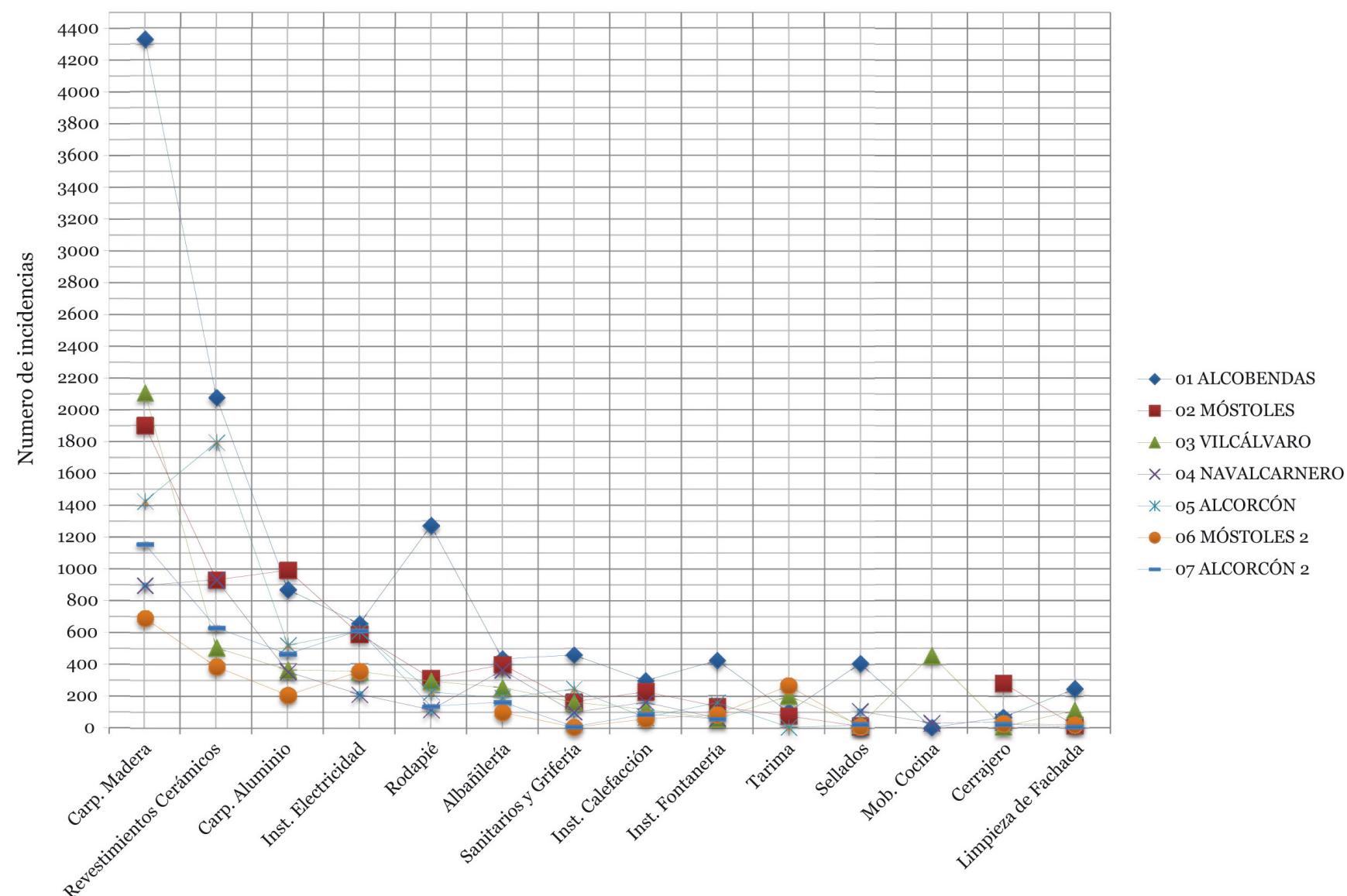

Oficio responsable de su reparación

Figura 3. Número de Incidencias por Oficios de Construcción. Comparativa de las 7 obras. 
unidad de obra, ya que una vez realizadas las reparaciones del resto de oficios se procede a emplastecer y lijar las zonas golpeadas, rozadas o con irregularidades de planeidad, y posteriormente se ejecuta la última mano de pintura de forma homogénea en toda la vivienda, evitando los defectos provocados por reparaciones puntuales en las que se suele apreciar las zonas reparadas por diferencia en el tono o textura de la pintura, y el sobre coste de realizar la segunda mano de pintura dos veces.

- Limpieza de Obra: dado que habitualmente la constructora procede a realizar de nuevo la limpieza de obra antes de presentar las viviendas a sus propietarios, se decide sacar este oficio del estudio, ya que ya se encuentra implementado en los protocolos de la empresa ésta acción.

También se decide suprimir del estudio todos los oficios cuyo montante de incidencias no supere el $1 \%$ del total. «A menudo los dos o tres primeros tipos de defectos suponen como mínimo el setenta u ochenta por cien del total. Está claro que si eliminamos estos defectos concretos, habremos eliminado la mayoría de los defectos, y la fracción de unidades defectuosa disminuirá espectacularmente» (5) (17). Para este artículo se resumen los datos de los diferentes histogramas en la Figura 3.

Con los datos modificados eliminando las incidencias de los oficios de Pintura, Limpieza de Obra y todos los que no alcanzan el 1\% del total de las incidencias, se elaboran nuevos diagramas de Pareto, con objeto de determinar qué oficios concentran el 80\% de las incidencias.
Como se aprecia en el diagrama de Pareto modificado del total de las 7 obras que se presenta en la Figura 4, los oficios en los que es preciso centrar los esfuerzos para conseguir resultados significativos son los siguientes:

- Carpintería de Madera

- Revestimientos Cerámicos

- Carpintería de Aluminio

- Instalación de Electricidad

- Rodapié

Los cuatro primeros oficios siguen siendo los mismos que se pusieron de manifiesto con los anteriores análisis, y se ha añadido el oficio de Rodapié.

En el diagrama de Pareto modificado que se presenta en la Figura 4 se ha marcado con dos líneas de color rojo la ordenada correspondiente al $80 \%$ del volumen acumulado de incidencias y la abscisa que marca el oficio límite del grupo que acumula el 80\% de los defectos.

Se aprecia al analizar las diferentes obras individualmente que los oficios que concentran al 80\% de las incidencias son bastante homogéneos, como se puede apreciar en el resumen presentado en la Tabla 4, a pesar de que en alguna obra de forma particular aparezca algún otro oficio dentro de este grupo.

De los cinco oficios que concentran el $80 \%$ de los defectos en el conjunto de las siete obras, Figura 4, sólo tres de ellos se encuentran también en este grupo en las siete obras de forma independiente:

Gráfico de Pareto - Número de Defectos - Total 7 Obras Fase 1

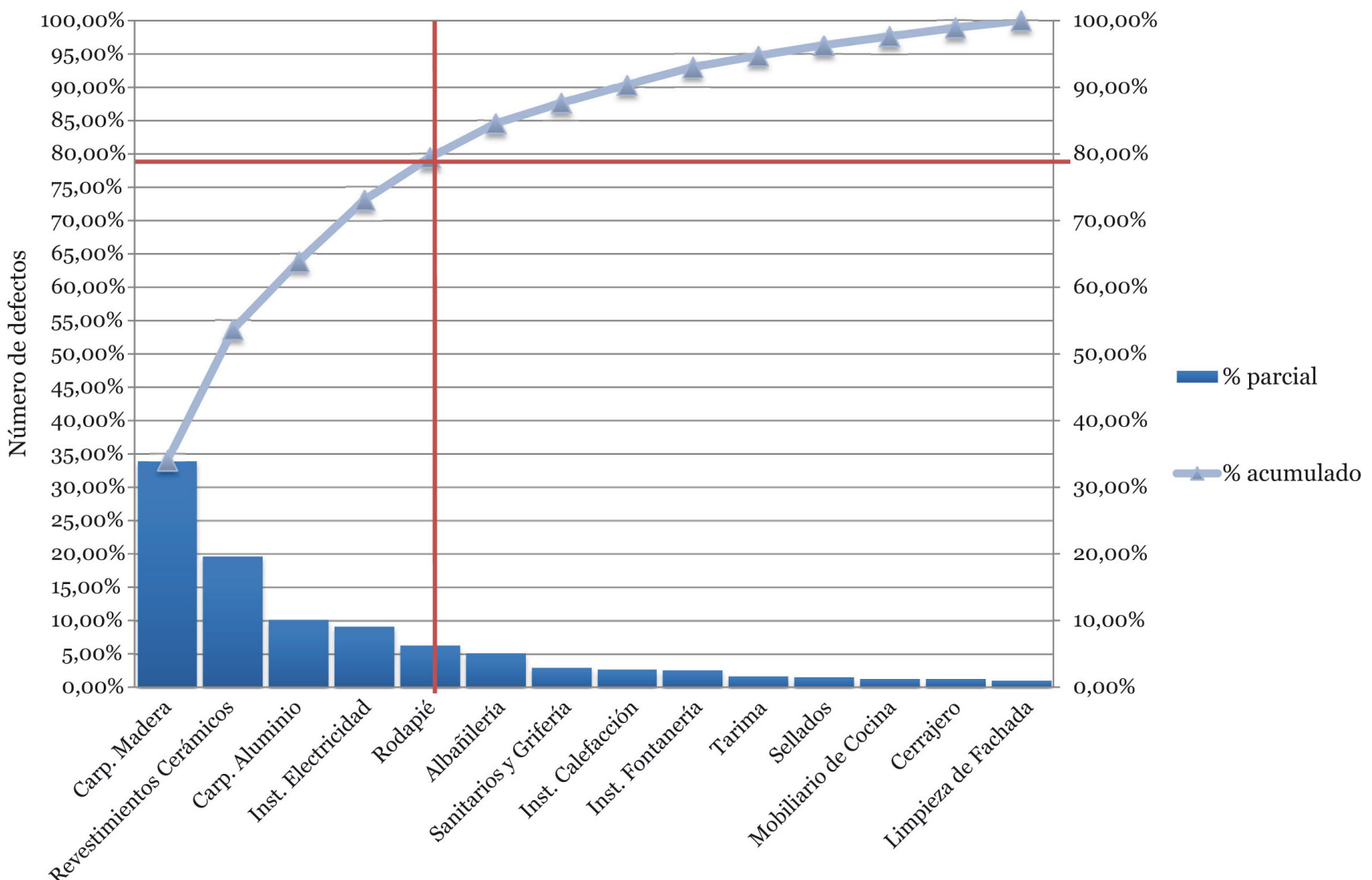

Figura 4. Diagrama de Pareto Modificado - Número de defectos por Oficio - Total 7 obras Fase 1. 
Tabla 4. Oficios que concentran el 80\% de las incidencias conforme a los diagramas de Pareto modificados.

\begin{tabular}{|l|c|c|c|c|c|c|c|c|}
\hline Oficio & Total & $\mathbf{0 1}$ & $\mathbf{0 2}$ & $\mathbf{0 3}$ & $\mathbf{0 4}$ & $\mathbf{0 5}$ & $\mathbf{0 6}$ & $\mathbf{0 7}$ \\
\hline Carpintería de Madera & $\mathrm{X}$ & $\mathrm{X}$ & $\mathrm{X}$ & $\mathrm{X}$ & $\mathrm{X}$ & $\mathrm{X}$ & $\mathrm{X}$ & $\mathrm{X}$ \\
\hline Revestimientos Cerámicos & $\mathrm{X}$ & $\mathrm{X}$ & $\mathrm{X}$ & $\mathrm{X}$ & $\mathrm{X}$ & $\mathrm{X}$ & $\mathrm{X}$ & $\mathrm{X}$ \\
\hline Carpintería de Aluminio & $\mathrm{X}$ & $\mathrm{X}$ & $\mathrm{X}$ & $\mathrm{X}$ & $\mathrm{X}$ & $\mathrm{X}$ & & \\
\hline Instalación de Electricidad & $\mathrm{X}$ & $\mathrm{X}$ & $\mathrm{X}$ & $\mathrm{X}$ & $\mathrm{X}$ & $\mathrm{X}$ & $\mathrm{X}$ & $\mathrm{X}$ \\
\hline Rodapié & $\mathrm{X}$ & $\mathrm{X}$ & $\mathrm{X}$ & $\mathrm{X}$ & & & & \\
\hline Albañilería & & & $\mathrm{X}$ & & $\mathrm{X}$ & & & \\
\hline Mobiliario de Cocina & & & & $\mathrm{X}$ & & & & \\
\hline Tarima & & & & & & & $\mathrm{X}$ & \\
\hline
\end{tabular}

- Carpintería de Madera

- Revestimientos Cerámicos

- Instalación de Electricidad

\section{CONCLUSIONES}

La Mejora Continua posibilita reducir los defectos de construcción y una de las posibles opciones es aplicar el Círculo de Calidad de Deming (PDCA). Para ello es preciso partir de datos suficientes para poder analizar donde se concentran los fallos y establecer, con la ayuda de las herramientas estadísticas contempladas en este estudio, las prioridades para poder acometer acciones encaminadas a la disminución significativa de los fallos.
Se ha podido determinar cuales son los tres oficios con más concentración de fallos: Carpintería de Madera, Revestimientos Cerámicos e Instalación de Electricidad. Si se analiza al detalle la causa de cada una de las incidencias será posible establecer las medidas necesarias para reducir drásticamente los costes de la no calidad en la construcción de viviendas.

El ejemplo de metodología simplificada para la Planificación de un Proyecto de Mejora desarrollada en este artículo (Hoja de recogida de datos, Estratificación, Histograma y Diagrama de Pareto) ayudará a los técnicos que intervienen en el proceso a realizar sus acciones de inspección más objetivas a la vez que posibilita la estandarización del proceso de estudio de los defectos.

\section{REFERENCIAS}

(1) García-Meseguer, A. (2001). Fundamentos de calidad en construcción. Sevilla: Fundación Cultural del Colegio Oficial de Aparejadores y Arquitectos Técnicos de Sevilla.

(2) AENOR. (2004). UNE-66178:2004. Sistemas de gestión de la calidad. Guía para la gestión del proceso de mejora continua. Madrid: AENOR.

(3) Garrido-Hernández, A. (1996). Aseguramiento de la calidad en la construcción. ICCE.

(4) Ishikawa, K. (1985). ¿Qué es el control total de calidad? La modalidad japonesa. NORMA S.A. (Traducción al castellano 1988).

(5) Membrado-Martínez, J. (2002). Innovación y mejora continua según el modelo EFQM de excelencia. Madrid: Ediciones Díaz de Santos.

(6) Chew, M.Y.L. (1999). Factors affecting ceramic tile adhesion for external cladding. Construction and Building Materials. 13(5): 293-296, doi: http://dx.doi.org/10.1016/So950-0618(99)00023-9.

(7) Chew, M.Y.L. (2005). Defects analysis in wet areas of buildings. Construction and Building Materials, 19(3): 165-173, doi: http://dx.doi.org/10.1016/j.conbuildmat.2004.07.005.

(8) Gardez, N., Lopes, N., Brito, J.de, Sá, G. (2012). System of inspection, diagnosis and repair of external claddings of pitched roofs. Construction and Building Materials, 35: 1034-1044, doi: http://dx.doi.org/10.1016/j.conbuildmat.2012.06.047.

(9) Garcez, N., Lopes, N., Brito, J.de, Sá, G. (2012). Pathology, diagnosis and repair of pitched roofs ceramic tiles. Construction and Building Materials. 36: 807-819, doi: http://dx.doi.org/10.1016/j.conbuildmat.2012.06.049.

(10) Neto, N., de Brito, J. (2012). Validation of an inspection and diagnosis system for anomalies in natural stone cladding (NSC). Construction and Building Materials, 30: 224-236, doi: http://dx.doi.org/10.1016/j.conbuildmat.2011.12.032.

(11) Pereida, A., Palha, F., de Brito, J., Silvestre, J.D. (2011). Inspection and diagnosis system for gypsum platers in partition walls and ceilings. Construction and Building Materials. 25(4): 2146-2156, doi: http://dx.doi.org/10.1016/j.conbuildmat.2010.11.015.

(12) Silvestre, J.D., de Brito, J. (2009). Ceramic tiling inspection system. Construction and Building Materials, 23(2): 653668, doi: http://dx.doi.org/10.1016/j.conbuildmat.2008.02.007.

(13) Silvestre, J.D., de Brito, J. (2011). Ceramic tiling in building façades: Inspection and pathological characterization using an expert system. Construction and Building Materials, 25(4): 1560-1571, doi: http://dx.doi.org/10.1016/j.conbuildmat.2010.09.039.

(14) Silva, A., de Brito, J., Gaspar, P.L. (2011). Service life prediction model applied to natural stone wall claddings (directly adhered to the substrate). Construction and Building Materials, 25(9): 3674-3684, doi: http://dx.doi.org/10.1016/j. conbuildmat.2011.03.064. 
(15) Rodrigues, M.F.S., Teixeira, J.M.C., Cardoso, J.C.P. (2011). Building envelope anomalies: A visual survey methodology. Construction and Building Materials, 25(6): 2741-2750, doi: http://dx.doi.org/10.1016/j.conbuildmat.2010.12.029.

(16) Macarulla, M., Forcada, N., Casals, M., Gangolells, M., Fuentes, A., Roca, X. (2013). Standardizing Housing Defects: Classification, Validation, and Benefits. Journal of Construction Engineering and Management, 139(8): 968-976, doi: http://dx.doi.org/10.1061/(ASCE)CO.1943-7862.0000669.

(17) Ishikawa, K. (1989). Introducción al control de calidad. Madrid: Ediciones Díaz de Santos.

(18) Galgano, A. (1995). Los siete instrumentos de la Calidad Total. Madrid: Ediciones Díaz de Santos.

(19) Chang, R., Niezwiecki, M.E. (1999). Las herramientas para la mejora continua de la calidad. (Volúmenes 1 y 2). Barcelona: Ed. Gránica. 